\title{
Numerical analysis of integrated photonics structures for hemoglobin sensor application
}

\author{
Przemysław Struk \\ Department of Optoelectronics, Faculty of Electrical Engineering, Silesian University of Technology, \\ 2 Krzywoustego Str. 44-100 Gliwice, Poland
}

Received May 13, 2020; accepted June 21, 2020; published June 30, 2020

\begin{abstract}
This paper presents a sensor structure dedicated for determining the key properties of a hemoglobin concentration level and oxidation level. The sensor structure is based on an integrated optics circuit with a diamond-based planar waveguide including a prism and a grating coupler. The paper is focused on numerical analysis of selected properties of sensor structure for optimization sensing parameters.
\end{abstract}

Modern medicine requires fast, accurate and costeffective sensors for determining the physical properties of biological liquids. One of the most important biological liquids in living organisms is blood. Blood is responsible for the transport of oxygen and nutrients to all body cells $[1 \div 2]$. In addition, blood is responsible for removing waste products and carbon dioxide from body cells. One of the key properties of blood is the oxidation level and hemoglobin concentration $[1 \div 3]$. An interesting group of sensors, which could be applied for determining the oxidation level and hemoglobin concentration are integrated optics-based structures $[4 \div 6]$. The integrated optics sensors structures offer a high scale of integration, high accuracy, low-cost, and short time of response

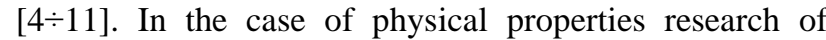
biological liquids, special attention is focused on sensors based on a planar waveguide equipped with grating couplers $[4 \div 12]$.

The paper presents a numerical analysis of selected components of the sensor structures based on a hybrid integrated optics circuit, which contain a prism coupler, planar waveguide, and grating coupler. The presented sensor is intended for determining the physical properties of blood such as oxidation level and hemoglobin concentration.

The hemoglobin sensor structure is built in a hybrid integrated optics circuit, which consists of three core components: prism coupler, planar waveguide, and grating coupler. The scheme of the hemoglobin sensor structure is presented in Fig. 1. The principle of operation of the hemoglobin sensor is the following: the prism coupler is responsible for the introduction of light from a laser into a planar waveguide structure. The planar waveguide (between the prism coupler and the grating coupler area) is responsible for the detection of a hemoglobin oxidation level by evanescence field. The evanescence field of the waveguide mode penetrates the hemoglobin cover layer. The changes in the hemoglobin oxidation level cause changes in an imaginary part of the refractive index $k$ of hemoglobin and hence changes in light attenuation of the planar waveguide. The changes of light attenuation are detected by a photodetector. A detailed numerical analysis of this part of sensor structure with a planar waveguide was presented by Author in papers [6]. The grating coupler in the sensor performs two functions. The first function - the grating coupler is responsible for the detection of hemoglobin concentration. The refractive index of hemoglobin $n_{c H}$ is dependent on concentration. Changes of hemoglobin concentration have influence on refractive index $\mathrm{n}_{\mathrm{ch}}$ and hence on effective refractive index $\mathrm{N}_{\text {eff }}$. The changes of effective refractive index $\mathrm{N}_{\text {eff }}$ cause changes of angle $\alpha$, for which the light is uncoupled from the structure. The principle of operation of sensors with grating coupler is described by equation $[6,7,13]$ :

$$
\alpha=\arcsin \left[\frac{1}{n_{c}}\left(N_{e f f}-\frac{m_{d} \lambda}{\Lambda}\right)\right],
$$

where: $\alpha-$ the angle of uncoupling light beam, $\Lambda-$ the spatial period, $n_{c}-$ the refractive index of cladding, $N_{\text {eff }}-$ the effective refractive index, $m_{d}-$ the diffraction order, $\lambda$ - the wavelength of light.

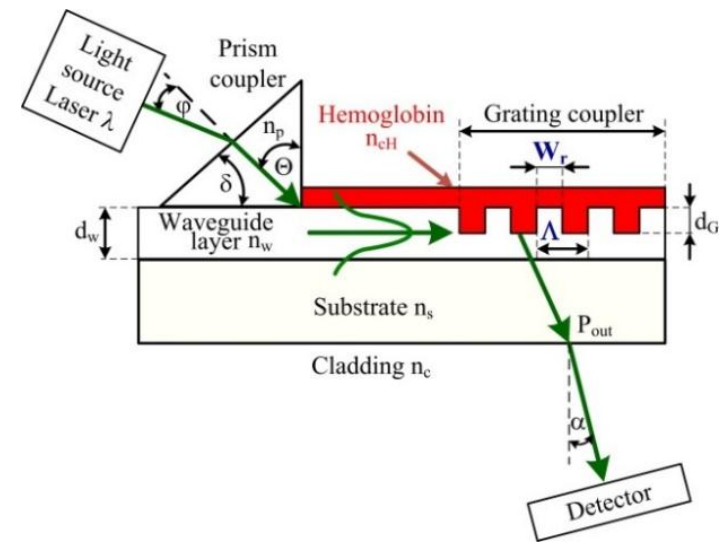

Fig. 1. Scheme of the haemoglobin sensor structure. 
The second function of the grating coupler in the presented sensor structure is the uncoupling of light from the sensor structure to the environment and hence to the detector. The key issue is the optimization of physical properties of the grating coupler, in particular, its geometrical properties such as: spatial period $\Lambda$, depth of grooves $d_{G}$ and duty cycle $k_{w}$.

The numerical analysis was divided into three parts. The first part was focused on the optimization of an optical and geometrical part of the sensor in the waveguide area (between the prism and the grating coupler). The results of the analysis such as: modal characteristic, homogeneous sensitivity, modal field distribution and propagation of light in the waveguide area with a hemoglobin cover layer were presented by Author in the previous paper [6]. The optimization of selected physical properties of the sensor in the area of the grating coupler - groove depth $\mathrm{d}_{\mathrm{G}}$ was presented in Ref. [12].

The numerical analysis presented in this paper was focused on the optimization of the duty cycle $k_{w}$ of the spatial period $\Lambda$ in the grating coupler. The duty cycle is determined as a width of the ridge $W_{r}$ to the spatial period $\Lambda$ in the grating coupler and described by equation:

$$
\mathrm{k}_{\mathrm{w}}=\frac{\mathrm{W}_{\mathrm{r}}}{\Lambda}
$$

where: $k_{w}$ - the duty cycle, $\Lambda$ - the spatial period of the grating coupler, $W_{r}$ - the width of the ridge in the grating coupler.

The optimal value of the duty cycle $k_{w}$ ensures the highest efficiency uncoupling of a light beam from the structure to the cladding, and hence to the detector, by the grating coupler. The numerical analysis was carried out for the following values of refractive indices: hemoglobin layer $n_{c H}=1.3681$ (concentration $26 \mathrm{~g} / \mathrm{dL}$ ), waveguide layer (diamond): $n_{w}=2.3$, substrate (quartz) $n_{s}=1.456$, cladding (air) $n_{c}=1[6,13,14]$. The grating coupler has a number of periods $P_{n}=25$, spatial period $\Lambda=0.6 \mu \mathrm{m}$ and depth of grooves $d_{G}=40 \mathrm{~nm}$. The waveguide layer thickness is $d_{w}=188 \mathrm{~nm}$. The analysis was carried out for the wavelength $\lambda=560 \mathrm{~nm}$. The numerical analysis was carried out for the selected waveguide mode - TE00. The waveguide mode was chosen as a compromise between the sensitivity of the structure presented in paper [6] and the possibility of practical realization of the sensor structure with a grating coupler with assumed geometrical properties (waveguide thickness, spatial period, depth of grooves and duty cycle).

The numerical analysis was carried out by using the Finite Difference Time Domain (FDTD) method and dedicated software OptiFDTD 32 Bit (Optiwave,
Canada). The numerical analysis was carried out for the boundary condition, which ensures the stability of the FDTD method - Courant-Friedrichs-Levy (CFL) [13, 15, 16]. The CFL condition is met for the following assumptions: mesh size on $x$-axis $\Delta x=0.002 \mu \mathrm{m}$, mesh size on $z$-axis $\Delta z=0.02 \mu \mathrm{m}$ and time step $\Delta t=4.4 \cdot 10^{-018} \mathrm{~s}$. On the edge of the modeled area was applied an anisotropic perfectly matched layer (APML).

The optimization of the duty cycle in the grating coupler was carried out in the range from $k_{w}=0$ to $k_{w}=1$ with the step $\Delta k_{w}=0.025$. The scheme of the grating coupler with the duty cycle $k_{w}=0.1, k_{w}=0.4, k_{w}=0.8$ is presented in Figs. $2 \mathrm{a} \div \mathrm{c}$, respectively.
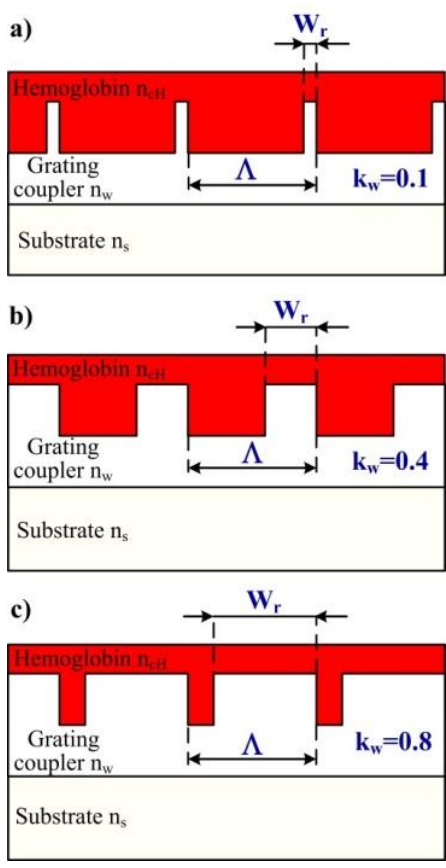

Fig. 2. Scheme of the analysed grating coupler with the duty cycle: a) $k_{w}=0.1$, b) $k_{w}=0.4, k_{w}=0.8$.

The first step of analysis was the determination of a modal field for TE00 mode in the waveguide structure (Fig. 3). This modal field was applied to optimize the duty cycle in the grating coupler.

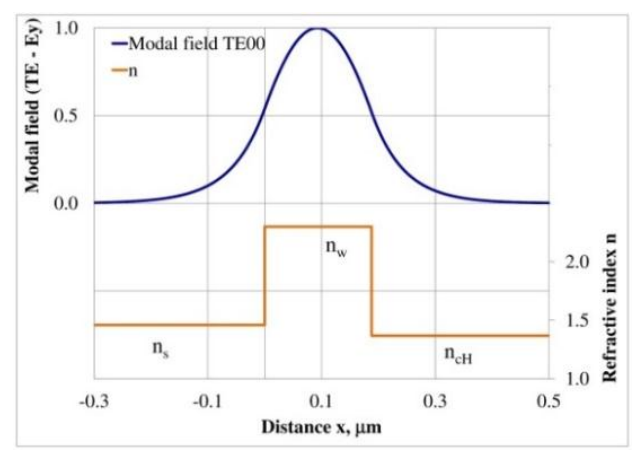

Fig. 3. Modal field distribution for TE00 mode. 
The presented results of numerical analysis show the influence of the duty cycle $k_{w}$ on grating coupler efficiency for uncoupling the light from the structure to the environment. The optical power of the light uncoupled by the grating couple as a function of the duty cycle $k_{w}$ for TE00 mode is presented in Fig. 4. If the duty cycle is increased, the optical power of the light uncoupled from the sensor structure to the environment via a grid coupler also increases until it reaches the maximum for $k_{w}=0.4$. If the duty cycle is still increased above the optimal value, the efficiency of the grating coupler decreases.

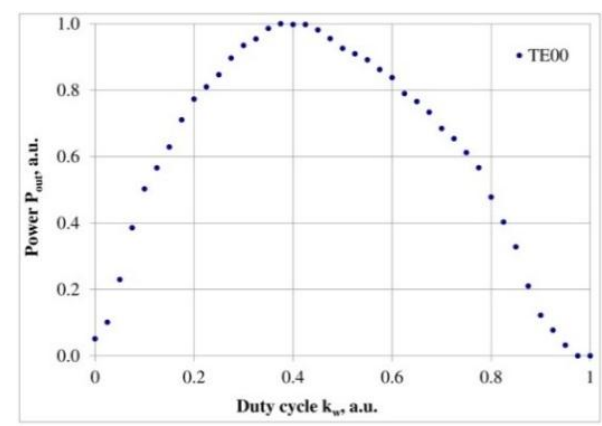

Fig. 4. Optical power uncoupled to detector as a function of the duty cycle for TE00 mode.

The Poynting vector for TE00 mode in the grating coupler area for the duty cycle $k_{w}=0.1, k_{w}=0.4$ and $k_{w}=0.8$ is presented in Figs. 5 $\div$, respectively.

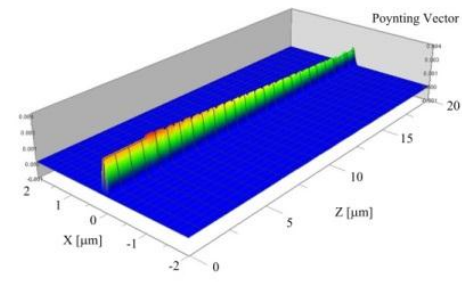

Fig. 5. Poynting vector in the sensor structure for $k_{w}=0.1, d_{w}=188 \mathrm{~nm}$, mode TE00.

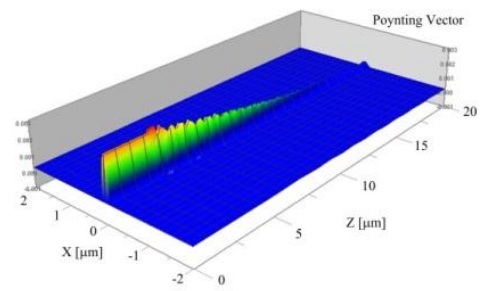

Fig. 6. Poynting vector in the sensor structure for $k_{w}=0.4, d_{w}=188 \mathrm{~nm}$, mode TE00.

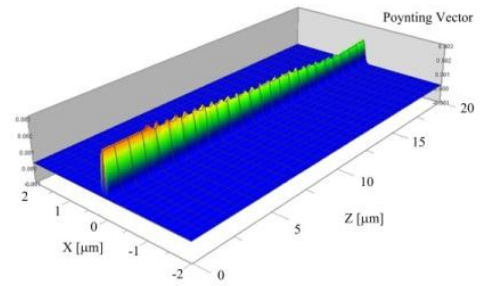

Fig. 7. Poynting vector in the sensor structure for $k_{w}=0.8, d_{w}=188 \mathrm{~nm}$ mode TE00.
According the numerical analysis presented above, the optimal value of the duty cycle for the spatial period $\Lambda=0.6 \mu \mathrm{m}$ in the grating coupler is $k_{w}=0.4$. In this case, the width of ridge is $W_{r}=0.24 \mu \mathrm{m}$ and hence the width of grooves $W_{g}=0.36 \mu \mathrm{m}$. In this case, the efficiency of the grating coupler for uncoupling of waveguide mode TE00 from the sensor structure to the environment is the highest.

In conclusions, the presented hemoglobin sensor structure is intended for determination of hemoglobin physical properties such as concentration and oxidation levels. The proper operation of the sensor required the optimization of optical and geometrical properties of the structure. The presented numerical analysis showed the relationship between the duty cycle of the spatial period and efficiency of the grating coupler. In the presented sensor structure, the optimal duty cycle ensuring the maximum efficiency of the grating coupler is at a level of $k_{w}=0.4$. The numerical analysis of optical and geometrical parameters of the sensor allows us to fabricate the structure with the highest sensing properties.

This study was partially financed by the pro-quality grant 05/040/RGJ20/2003 - Silesian University of Technology.

\section{References}

[1] D.A. Gell, Blood Cells, Molecules and Diseases 70, 13(2018)

[2] I. Singh, A. Weston, A. Kundur, G. Dobie, Haematology Case Studies with Blood Cell Morphology and Pathophysiology (Elsevier: Amsterdam, The Netherlands, 2017).

[3] P.D. Sturkie, P. Griminger, Blood: Physical Characteristics, Formed Elements, Hemoglobin, and Coagulation Avian Physiology (Springer-Verlag New York Inc. 1976).

[4] A. Dutta, B. Deka, P.P. Partha, Planar Waveguide Optical Sensors: From Theory to Applications (Springer: Berlin, Germany, 2016).

[5] S. Kang, K. Sasaki, H. Minamitani, IEEE Engineering in Medicine and Biology Society (1992).

[6] P. Struk, Materials 12(1), 175 (2019).

[7] W. Lukosz, K. Tiefenthaler, Sensors and Actuators 15, 211(1988).

[8] P.V. Lambeck, Measurement Science and Technology - IOP Science 17(8), R93 (2006),

[9] W. Lukosz, Sensors and Actuators B: Chemical 29, 37( 1995).

[10] P. Struk, T. Pustelny, K. Gołaszewska, E. Kamińska, M. Borysiewicz, M. Ekielski, And A. Piotrowska, Opto-Electr. Rev. 19(4), 462 (2011).

[11] J. Haas, E. V. Catalán, P. Piron, F. Nikolajeff, L. Österlund, M. Karlsson, B. Mizaikoff, ACS Omega 3, 6190 (2018).

[12] P. Struk, Proceedings of SPIE 11204, 1120401 (2019).

[13] P. Struk, T. Pustelny, K. Gołaszewska, E. Kamińska, M. Borysiewicz, M. Ekielski, A. Piotrowska, Opto-Electr. Rev. 21, 376 (2013).

[14] V. Prajzler, M. Varga, P. Nekvindova, Z.; Remes, A. Kromka, Opt. Expr. 21, 8417 (2013).

[15] E.N. Lazareva, V.V. Tuchin, J. Biomedical Optics 23, 035004 (2018).

[16] Optiwave Systems Inc. OptiFDTD Technical Background and Tutorials - Finite (2013).

[17] K. Yee, IEEE Transactions 14, 3 (1966). 